\title{
A influência da rede de relações, do coping e do neuroticismo na satisfação de vida de jovens estudantes
}

\author{
The influence of the network of relationships, \\ coping and neuroticism on the life \\ satisfaction of young students
}

\author{
Adriana Jung SERAFINI ${ }^{1,2}$ \\ Denise Ruschel BANDEIRA ${ }^{3}$
}

\begin{abstract}
Resumo
Objetivou-se avaliar, em uma amostra de jovens, o efeito das variáveis rede de relações, neuroticismo e estratégias de coping sobre a variável satisfação de vida, e apresentar um perfil descritivo da amostra para essas variáveis. Participaram 502 alunos de 10 escolas estaduais de Porto Alegre, com idade média de 16,6 anos (desvio-padrão=1,4 anos). Os instrumentos utilizados incluem: questionário de dados sociodemográficos, Escala Multidimensional de Satisfação de Vida para Adolescentes, Inventário de Rede de Relações, Inventário de Estratégias de Coping e Escala Fatorial de Neuroticismo. Foram realizadas análises de variância multivariada e de regressão múltipla. Os principais resultados indicaram que os participantes apresentam bom nível de satisfação de vida, utilizam um número variado de estratégias de coping, e que os amigos e a mãe exercem influência sobre eles. Verificou-se que o fator depressão da Escala Fatorial de Neuroticismo foi o que melhor predisse o nível de satisfação de vida total da amostra.
\end{abstract}

Unitermos: Coping. Jovens. Neuroticismo. Relações interpessoais. Satisfação de vida.

\begin{abstract}
The aim of this study was to assess if the variables "network relationships", "neuroticism" and "coping strategies" would have an effect on the variable "ife satisfaction", in a sample of young people, and to present a descriptive profile of the sample for each of the variables. A total of 502 students, from 10 state schools in Porto Alegre, Brazil, took part in this study. The average age was 16.6 years (standard deviation = 1.4years). The instruments used comprised: a socio-demographic data questionnaire, Multidimensional Life Satisfaction Scalefor Adolescents, Network Relationships Inventory, Coping Strategies Inventory and Neuroticism Factorial Scale. A multivariate variance analysis and linear multiple regression were carried out. The most significant results showed that the participants demonstrated a good level of life satisfaction, used a variety of coping strategies and that friends and mothers seemed to exert an influence on them. The best predictor for the level of Total Life Satisfaction was the factor Depression in the Neuroticism Factorial Scale.
\end{abstract}

Uniterms: Coping. Youth. Neuroticism. Interpersonal relationships. Life satisfaction.

$\operatorname{ratr}$

1 Universidade Federal do Rio Grande do Sul, Instituto de Psicologia. R. Ramiro Barcelos, 2600, Sala 120, 90035-903, Porto Alegre, RS, Brasil. Correspondência para/Correspondence to: A.J. SERAFINI. E-mail: <adrianaserafini@terra.com.br>.

2 Universidade Federal de Ciências da Saúde de Porto Alegre, Departamento de Psicologia. Porto Alegre, RS, Brasil.

3 Universidade Federal do Rio Grande do Sul, Curso de Psicologia. Porto Alegre, RS, Brasil.

Apoio: Conselho Nacional de Desenvolvimento Científico e Tecnológico e a Coordenação de Aperfeiçoamento de Pessoal de Nível Superior. 
A satisfação de vida é considerada o elemento cognitivo que constitui o bem-estar subjetivo. Ela é definida como uma avaliação global que a pessoa faz de sua própria vida. Essa avaliação poderia ser descrita como uma comparação que as pessoas realizam das situações que experenciaram, de acordo com um padrão construído por elas mesmas (Pavot, Diener, Colvin \& Sandivik, 1991). Além dessa avaliação global de satisfação de vida, Hueber (1991) enfatiza a importância de um julgamento a partir de domínios específicos, como, por exemplo, a família ou os amigos.

Como a presente pesquisa refere-se a uma população específica - os jovens -, torna-se importante abordar estudos sobre satisfação de vida de indivíduos nessa faixa etária. Os resultados demonstram, de forma geral, que os jovens apresentam um nível alto de satisfação de vida (Nickerson \& Nagle, 2004). Apesar disso, pesquisas indicam que certas variáveis podem influenciar o nível de bem-estar, e, como consequência, o nível de satisfação. Entre essas variáveis estariam: traços de personalidade, eventos de vida, estratégias de coping e presença de uma rede de relações (Aldwin \& Revenson, 1987; Diener \& Lucas, 1999; Nickerson \& Nagle, 2004).

Um dos achados mais consistentes no campo do bem-estar subjetivo diz respeito ao fato de seus componentes estarem moderadamente relacionados a traços de personalidade, caracterizados como preditores de felicidade. Pesquisas relacionando o modelo de personalidade dos Cinco Grandes Fatores indicam que os traços de personalidade mais fortemente relacionados ao bem-estar subjetivo são a extroversão e o neuroticismo (Diener \& Lucas, 1999; Hayes \& Joseph, 2003).

Apesar desses achados alusivos à personalidade, alguns pesquisadores fazem referência à importância exercida pelo impacto de certos eventos de vida sobre o bem-estar (Diener \& Lucas, 1999). Em função de tais influências, o bem-estar subjetivo poderia ser considerado não apenas como um traço, mas também como estado (Feist, Bodner, Jacobs, Miles \& Tan, 1995).

Quando se fala em eventos de vida, é importante que se investigue as formas como a pessoa lida com esses eventos, ou seja, avaliar as estratégias de coping. Coping é definido por Lazarus e Folkman (1984) como os esforços cognitivos e comportamentais para lidar com demandas externas e/ou internas específicas que são avaliadas como excedendo os esforços e recursos do indivíduo. A importância de se estudarem as estratégias de coping está no fato de que sua efetividade relaciona-se com resultados positivos sobre aspectos da saúde física ou mental das pessoas. Assim, podemos entender sua efetividade também relacionada à satisfação de vida (Aldwin \& Revenson, 1987).

Resultados de pesquisas demonstram que outro fator que exerce influência sobre o bem-estar de adolescentes é o suporte social advindo de relações interpessoais positivas (Huebner, 2004). Ainda no que se refere às relações interpessoais, estudos brasileiros, de forma geral, demonstram que essas relações são significativamente marcantes durante a adolescência, e que as relações entre amigos da mesma idade possuem um papel importante para o desenvolvimento da personalidade e para o ajustamento dos adolescentes. Juntamente com amigos, pais e irmãos demonstraram ser as fontes mais importantes de apoio, não havendo distinção entre eles (Marques, 1996; Marques \& Horn, 2002).

Como visto na revisão teórica, percebe-se que nas últimas décadas tem crescido a necessidade de os pesquisadores e teóricos avaliarem aspectos relacionados a elementos positivos na avaliação do bem-estar e da saúde do homem. Entretanto, pesquisas brasileiras que avaliem conjuntamente o efeito de características de personalidade, da rede de relações e das estratégias de coping sobre a satisfação nessa fase da vida não foram encontradas nas bases indexadas SciELO, Lilacs e Psyclnfo. Desse modo, este estudo tem como objetivo geral verificar se existe efeito das variáveis rede de relações, personalidade e coping sobre a satisfação de vida dos adolescentes. Como objetivo específico, busca-se apresentar um perfil descritivo da amostra, investigando possíveis diferenças entre sexos para as seguintes variáveis: satisfação de vida, relações estabelecidas pelos jovens, estratégias de coping e características de personalidade.

\section{Método}

\section{Participantes}

Participou deste estudo uma amostra aleatória por conglomerados composta por 502 alunos do ensino médio, provenientes de 10 escolas da rede estadual 
situadas em oito bairros da cidade de Porto Alegre (RS). Dos participantes, 54,2\% eram do sexo feminino, e a idade variou entre 14 e 23 anos (Média-M=16,6, Desvio-Padrão - DP =1,4 anos).

O maior percentual dos jovens $(83,7 \%)$ estudava no turno diurno e encontrava-se cursando o primeiro ano do ensino médio (45,9\%), seguido pelos estudantes do segundo $(37,3 \%)$ e terceiro ano do ensino médio (16,7\%). A maior parte dos jovens pertencia às classes $C$ e B2, cujas rendas médias são de R\$927,00 e R\$1.669,00, respectivamente (Associação Brasileira de Empresas de Pesquisa - ABEP, 2003). No que se refere ao estado civil e às relações afetivas, 480 jovens (95,6\%) eram solteiros, e, destes, 192 (40,0\%) encontravam-se namorando.

\section{Instrumentos}

Foram aplicados os seguintes instrumentos (em ordem de aplicação):

- Questionário de Dados Sociodemográficos, que contém questões relativas à idade, sexo, escolaridade, dados dos pais (escolaridade, profissão), número de irmãos, estado civil, relacionamentos (como, por exemplo, se tem namorado(a), com que idade começou a namorar), entre outras. Há questões, ainda, sobre a experiência de certos eventos de vida, como o uso de drogas, presença de alguma doença grave, hospitalização, depressão, gravidez etc. A avaliação da classe social dos participantes foi realizada por meio do Critério de Classificação Econômica Brasil da Associação Brasileira de Empresas de Pesquisa (ABEP) (2003), presente no questionário.

- Escala Multidimensional de Satisfação de Vida para Adolescentes (EMSV-A) de Arteche (2003), uma adaptação da escala de Giacomoni e Hutz (2008), que avalia a satisfação de vida por meio de sete fatores: self, self-comparado, família, escola, não-violência, amizade e global). Seus 57 itens são respondidos por meio de uma escala Likert, com as seguintes opções de repostas: (1) nem um pouco, (2) bem pouco, (3) mais ou menos, (4) bastante e (5) muitíssimo.

- Inventário de Rede de Relações (IRR), de Furman e Buhrmester (1992), adaptado para o Brasil por Marques e Horn (2002), avalia 11 dimensões de relacionamentos interpessoais por meio de 33 itens. As dimensões englobadas pelo instrumento são conflito, poder relativo, punição, satisfação e sete dimensões de apoio social (companhia, auxílio instrumental, autorrevelação, cuidado com o outro, afeição, valorização e aliança confiável). Essas dimensões foram classificadas com cada uma das seguintes pessoas: mãe, pai, melhor amigo(a), namorado(a)/marido(esposa)/companheiro(a). As respostas são registradas em uma escala Likert de cinco pontos, que indica a qualidade de relacionamento com cada uma das pessoas citadas.

- Inventário de Estratégias de Coping de Folkman e Lazarus (1985), adaptado para o Brasil por Savóia, Santana e Mejitas (1996), que avalia estratégias de coping por meio de oito fatores: confronto, afastamento, autocontrole, suporte social, aceitação de responsabilidade, fuga-esquiva, resolução de problemas e reavaliação positiva. Seus 66 itens são respondidos pelo participante de acordo com a intensidade com que utilizaram cada estratégia mencionada, em determinada situação, tendo como opções de respostas: (0) não usei esta estratégia, (1) usei um pouco, (2) usei bastante e (3) usei em grande quantidade.

- Escala Fatorial de Ajustamento Emocional/ Neuroticismo (EFN) (Hutz, \& Nunes, 2001), que avalia a dimensão da personalidade humana denominada neuroticismo/estabilidade emocional (modelo dos cinco grandes fatores) por meio do levantamento de traços de personalidade. O teste é composto por 82 itens em quatro subescalas (escala de vulnerabilidade, escala de desajustamento psicossocial, escala de ansiedade e escala de depressão). Os itens são registrados em uma escala Likert de sete pontos, em que 1 ="a sentença não descreve nenhuma característica minha" e 7 = "a sentença me descreve perfeitamente bem".

\section{Procedimentos}

Foi obtida uma lista das escolas estaduais da rede pública de Porto Alegre, na Secretaria da Educação a partir da qual foi realizado um sorteio das escolas participantes. Após o sorteio, foi feito o contato com as escolas para obter o consentimento para a realização da pesquisa e para fazer o convite aos alunos. Os coordenadores das escolas indicaram as turmas que participariam, de acordo com a disponibilidade dos professores. Assim, foram privilegiadas as turmas que não tinham prova na data da coleta ou conteúdos que pudessem ser adiados. 
Antes da aplicação, os alunos foram informados sobre os procedimentos e objetivos do estudo. OTermo de Consentimento Livre e Esclarecido, após ser entregue, foi lido juntamente com os participantes. Após o esclarecimento de eventuais dúvidas, os instrumentos foram aplicados em sala de aula, de forma coletiva. Os materiais foram entregues todos em um único momento, grampeados. Antes do preenchimento, a pesquisadora, juntamente com um aluno de psicologia devidamente treinado, dava as instruções aos alunos. Aqueles que desejaram participar preenchiam o material. No caso de dúvidas, os aplicadores auxiliaram os participantes individualmente. O projeto de pesquisa teve aprovação do Comitê de Ética da escola de Saúde Pública da Secretaria de Saúde do Estado do Rio de Grande do Sul, protocolo no 091/2006.

Para fins de análise dos dados, foi utilizado o pacote estatístico Statistical Package for the Social Sciences (SPSS) versão 13.0. Na construção do banco de dados, as respostas referentes aos instrumentos EMSV-A, IRR, Inventário de Estratégias de Coping e EFN, que haviam sido deixadas em branco, foram substituídas pela média do grupo naquele item.

Os instrumentos foram avaliados do ponto de vista psicométrico, e os dados obtidos analisados por meio de estatísticas descritivas, sendo apresentadas médias e desvios-padrão das diversas medidas. Para responder ao objetivo específico, os dados dos instrumentos citados anteriormente foram analisados por meio de MANCOVA, sendo incluída como variável independente sexo. A variável idade foi utilizada como controle em função de ter sido encontrada correlação entre ela e diversos fatores dos instrumentos utilizados. No IRR, as análises foram realizadas com os 11 fatores do instrumento para cada uma das seguintes pessoas: mãe, pai e melhor amigo. Foram excluídos namorado(a)/ marido(esposa)/companheiro(a) e outro, pelo fato de poucos participantes (menos de 50\% e 25\% da amostra respectivamente) terem respondido para essas pessoas.

Por fim, para responder ao objetivo geral deste estudo, foi realizada uma análise de regressão múltipla. O teste estatístico teve como meta avaliar o efeito das variáveis independentes (fatores relacionados à personalidade, estratégias de coping e rede de relações) sobre a variável dependente (fatores relacionados à satisfação 18 de vida).
O projeto desta pesquisa foi aprovado pelo Comitê de Ética da Escola de Saúde Pública do Rio Grande do Sul. Antes da aplicação dos instrumentos, os jovens com idades a partir de 18 anos que aceitaram participar preencheram oTermo de Consentimento Livre e Esclarecido. Para aqueles com idade inferior a 18 anos, solicitou-se que o aluno e os responsáveis preenchessem o termo. $\mathrm{O}$ documento foi assinado em duas vias, uma permanecendo com a pesquisadora e a outra com o participante.

\section{Resultados}

Em relação aos seus aspectos psicométricos, a EMSV-A apresentou boa consistência interna $(\alpha=0,91)$. A Tabela 1 apresenta os resultados das análises das médias por sexo e faixa etária para todos os fatores da escala e sua soma total.

Como as respostas da EMSV-A podem variar de 1 a 5 , pode-se observar que os adolescentes da amostra apresentaram um bom nível de satisfação de vida. Todas as médias (para o total de jovens) situaram-se entre 3,5 e 4.

Os resultados da MANCOVA mostraram que na análise por sexo os grupos diferenciaram-se em dois fatores, o fator escola e o fator não-violência. No primeiro fator o grupo feminino apresentou uma média significativamente maior do que o grupo masculino. No segundo, o grupo masculino apresentou uma média significativamente maior .

Assim como a EMSV-A, o IRR também apresentou boa consistência interna. O inventário foi analisado separadamente para cada uma das pessoas classificadas: mãe $(\alpha=0,87)$, pai $(\alpha=0,93)$ e melhor amigo $(\alpha=0,93)$.

Após essa etapa, foram realizadas análises de covariância com os fatores que compõem o IRR para cada um dos relacionamentos citados anteriormente, tendo como variável independente sexo. Na Tabela 2, em função da grande quantidade de fatores, são apresentados somente os resultados cujas médias diferenciam-se entre os sexos.

Por meio da análise dos resultados, percebe-se que as maiores diferenças entre os sexos encontram-se nos fatores associados ao melhor amigo, seguido por 
pai e por último mãe. Verificam-se diferenças significativamente mais altas nas médias das meninas para os fatores necessidade de auxílio instrumental do meIhor amigo, revelação/intimidade com melhor amigo, relação de cuidado com melhor amigo, e afeição pelo melhor amigo. Além disso, percebe-se que o fator conflito com o melhor amigo apresenta média mais alta para os meninos do que para as meninas da amostra.

As médias dos participantes do sexo masculino mostraram-se significativamente mais altas do que as do sexo feminino nos fatores de satisfação na relação com o pai e necessidade de auxílio instrumental por parte dele. Apesar de as médias terem sido baixas para ambos os gêneros, o fator de revelação/intimidade com o pai também foi significativamente mais alto para meninos do que meninas. Percebe-se, também, que enquanto para as meninas a relação com a mãe parece exercer uma maior influência, com média significativamente mais alta no fator revelação/intimidade com a mãe, para os meninos o pai parece ser a figura de maior influência.

O Inventário de Estratégias de Coping apresentou uma boa consistência interna $(\alpha=0,93)$. A primeira etapa para o preenchimento do inventário consistia em citar uma situação de stress para a qual os participantes iriam classificar as estratégias utilizadas. As diversas situações relatadas pelos participantes foram agrupadas em 17 categorias elaboradas por meio de análise de conteúdo (Bardin, 1979), reunindo-se aquelas que se mostravam semelhantes. Observou-se que as situações mais citadas foram as relacionadas a problemas com pais, familiares, amigos ou parceiros(as) (31,87\%), problemas na escola ou no trabalho (15,13\%) e problemas cotidianos (10,55\%). Em percentuais bem mais baixos foram citadas si-

Tabela 1. Resultados da MANCOVA para os fatores da EMSV-A. Porto Alegre (RS), 2008.

\begin{tabular}{|c|c|c|c|c|c|c|c|}
\hline \multirow{3}{*}{ Fatores } & \multirow{2}{*}{\multicolumn{2}{|c|}{ Total $(n=502)$}} & \multicolumn{4}{|c|}{ Sexo } & \multirow{3}{*}{$\mathrm{F}$} \\
\hline & & & \multicolumn{2}{|c|}{ Feminino $(n=272)$} & \multicolumn{2}{|c|}{ Masculino $(n=230)$} & \\
\hline & M & DP & M & $\mathrm{DP}$ & $M$ & DP & \\
\hline Família & 4,05 & 0,59 & 4,04 & 0,60 & 4,05 & 0,59 & 0,02 \\
\hline Self comparado & 3,59 & 0,66 & 3,56 & 0,65 & 3,63 & 0,66 & 1,46 \\
\hline Escola & 3,48 & 0,66 & 3,58 & 0,65 & 3,35 & 0,65 & $15,72^{*}$ \\
\hline Não-violência & 3,86 & 0,71 & 3,74 & 0,72 & 3,99 & 0,67 & $14,46^{*}$ \\
\hline Amizade & 3,97 & 0,51 & 3,98 & 0,51 & 3,93 & 0,52 & 0,83 \\
\hline Self & 4,06 & 0,58 & 4,06 & 0,58 & 4,04 & 0,59 & 0,08 \\
\hline Satisfação de vida global & 3,96 & 0,71 & 3,91 & 0,70 & 4,01 & 0,72 & 2,24 \\
\hline Satisfação de vida total & 3,88 & 0,42 & 3,87 & 0,42 & 3,88 & 0,42 & 0,13 \\
\hline
\end{tabular}

*Diferença significativa entre médias, $p<0,01$.

M: média; DP: desvio-padrão; EMSV-A: escala multidimensional de satisfação de vida para adolescentes; Mancova: análise multivariada de covariância.

Tabela 2. Resultados da MANCOVA para os fatores do IRR. Porto Alegre (RS), 2008.

\begin{tabular}{|c|c|c|c|c|c|c|c|c|c|c|}
\hline \multirow{3}{*}{ Fatores } & \multirow{2}{*}{\multicolumn{3}{|c|}{ Total }} & \multicolumn{6}{|c|}{ Sexo } & \multirow{3}{*}{ F } \\
\hline & & & & \multicolumn{3}{|c|}{ Feminino } & \multicolumn{3}{|c|}{ Masculino } & \\
\hline & $n$ & M & $\mathrm{DP}$ & $n$ & $M$ & DP & $n$ & M & DP & \\
\hline Revelação mãe & 532 & 2,63 & 1,30 & 269 & 2,75 & 1,36 & 225 & 2,40 & 1,20 & $11,89^{*}$ \\
\hline Auxílio instrumental do pai & 476 & 2,87 & 1,19 & 243 & 2,73 & 1,18 & 207 & 2,96 & 1,19 & $4,52^{* *}$ \\
\hline Satisfação como pai & 476 & 3,50 & 1,26 & 243 & 3,30 & 1,21 & 207 & 3,65 & 1,29 & $7,26^{*}$ \\
\hline Revelação do pai & 476 & 1,80 & 0,96 & 243 & 1,58 & 0,78 & 207 & 1,89 & 0,98 & $15,12^{*}$ \\
\hline Conflito com o amigo & 514 & 1,90 & 0,79 & 259 & 1,78 & 0,67 & 220 & 1,92 & 0,75 & $5,84^{*}$ \\
\hline Auxílio instrumental do amigo & 499 & 3,17 & 0,95 & 259 & 3,32 & 0,90 & 220 & 2,98 & 0,96 & $15,92^{*}$ \\
\hline Revelação do amigo & 499 & 3,51 & 1,23 & 259 & 3,86 & 1,11 & 220 & 3,18 & 1,22 & $35,11^{*}$ \\
\hline Cuidado com amigo & 500 & 3,67 & 0,93 & 260 & 3,77 & 0,88 & 220 & 3,50 & 0,96 & $8,416^{*}$ \\
\hline Afeição do amigo & 499 & 3,90 & 0,90 & 259 & 4,01 & 0,87 & 220 & 3,69 & 0,90 & $13,84^{*}$ \\
\hline
\end{tabular}

*Diferença significativa entre médias, $p<0,01 ;{ }^{* *}$ Diferença significativa entre médias, $p<0,05$.

M: média; DP: desvio-padrão; Mancova: análise multivariada de covariância; IRR: inventário de rede de relações. 
tuações mais extremas e/ou de risco, como o uso de drogas/envolvimento com o tráfico de drogas (0,99\%), violência familiar (0,39\%) e ser preso (0,39\%).

Para a análise das estratégias utilizadas para lidar com a situação de estresse foi realizada uma MANCOVA, incluindo-se os oito fatores do instrumento (Tabela 3).

Percebe-se que a estratégia mais utilizada pelo grupo de jovens da amostra foi a de Suporte Social, com média de 1,37 (desvio-padrão de 0,69). No que se refere à variável sexo, observa-se que os jovens do sexo masculino apresentaram uma média significativamente mais alta no fator Afastamento.

Como nos demais instrumentos, a Escala Fatorial de Neuroticismo também apresentou uma consistência interna, com Alpha de Cronbach de 0,94. A análise entre médias utilizou os quatro fatores da escala e seus resultados são evidenciados na Tabela 4.

Os resultados demonstram diferenças significativas entre os sexos nas médias dos fatores ansiedade, desajustamento e depressão. no fator ansiedade, as jovens do sexo feminino apresentaram uma média significativamente mais alta, enquanto nos demais fatores os jovens do sexo masculino apresentaram médias mais altas.

Para verificar se existiria efeito entre as variáveis Rede de Relações, Personalidade e Coping sobre a satisfação de vida dos adolescentes, foi realizada uma análise de regressão linear multivariada por meio do método stepwise. Utilizaram-se como variáveis independentes as 11 dimensões do IRR analisadas para cada uma das seguintes pessoas: mãe, pai e melhor amigo. Os oito fatores do Inventário de Estratégias de Coping também foram utilizados como variáveis independentes, assim como as quatro subescalas da EFN. A variável dependente foi a Satisfação de Vida Total, obtida por meio da média de todos os itens da EMSV-A. A Tabela 5 apresenta os resultados da análise de regressão linear multivariada com os fatores que predisseram a satisfação de vida dos participantes da amostra.

Tabela 3. Resultados da MANCOVA para os fatores do Inventário de Estratégias de Coping. Porto Alegre (RS), 2008.

\begin{tabular}{|c|c|c|c|c|c|c|c|}
\hline \multirow{3}{*}{ Fatores } & \multirow{2}{*}{\multicolumn{2}{|c|}{ Total $(n=502)$}} & \multicolumn{4}{|c|}{ Sexo } & \multirow{3}{*}{$F(1,501)$} \\
\hline & & & \multicolumn{2}{|c|}{ Feminino $(n=272)$} & \multicolumn{2}{|c|}{ Masculino $(n=230)$} & \\
\hline & $M$ & $\mathrm{DP}$ & $M$ & $\mathrm{DP}$ & M & $\mathrm{DP}$ & \\
\hline Confronto & 0,63 & 1,21 & 0,60 & 1,22 & 1,19 & 0,66 & 0,32 \\
\hline Afastamento & 0,61 & 1,10 & 0,62 & 1,04 & 1,17 & 0,59 & $5,12^{*}$ \\
\hline Autocontrole & 0,60 & 1,26 & 0,60 & 1,23 & 1,30 & 0,60 & 1,20 \\
\hline Suporte social & 0,69 & 1,37 & 0,68 & 1,42 & 1,32 & 0,70 & 3,24 \\
\hline Aceitação de responsabilidade & 0,75 & 1,31 & 0,76 & 1,27 & 1,35 & 0,73 & 1,24 \\
\hline Fuga e esquiva & 0,61 & 1,04 & 0,59 & 1,06 & 1,03 & 0,64 & 0,40 \\
\hline Resolução de problemas & 0,68 & 1,25 & 0,68 & 1,19 & 1,31 & 0,68 & 3,12 \\
\hline Reavaliação positiva & 0,64 & 1,34 & 0,68 & 1,30 & 1,37 & 0,62 & 1,22 \\
\hline
\end{tabular}

*Diferença significativa entre médias, $p<0,01$.

M: média; DP: desvio-padrão.

Tabela 4. Resultados da MANCOVA para os fatores da escala fatorial de neuroticismo. Porto Alegre (RS), 2008.

\begin{tabular}{|c|c|c|c|c|c|}
\hline \multirow{3}{*}{ Fatores } & \multicolumn{4}{|c|}{ Sexo } & \multirow{3}{*}{$\mathrm{F}$} \\
\hline & \multicolumn{2}{|c|}{ Feminino $(n=272)$} & \multicolumn{2}{|c|}{ Masculino $(n=230)$} & \\
\hline & M & $\mathrm{DP}$ & $M$ & $\mathrm{DP}$ & \\
\hline Vulnerabilidade & 70,33 & 23,24 & 71,85 & 23,57 & 0,38 \\
\hline Ansiedade & 81,22 & 25,84 & 73,40 & 24,65 & $12,81^{*}$ \\
\hline Desajustamento & 27,19 & 12,48 & 32,87 & 13,50 & $22,42^{*}$ \\
\hline Depressão & 46,26 & 16,51 & 50,27 & 18,58 & $6,21^{*}$ \\
\hline
\end{tabular}

*Diferença entre médias significativa, $p<0,01$.

20 M: média; DP: desvio-padrão. 
Tabela 5. Resultados da análise de regressão linear multivariada. Porto Alegre (RS), 2008.

\begin{tabular}{lcccc}
\hline \multirow{2}{*}{ Variáveis Independentes } & \multicolumn{4}{c}{ Satisfação de vida total } \\
\cline { 2 - 4 } & $\beta$ & $R$ & $R^{2}$ & $R^{2}$ ajustado \\
\hline Depressão & $-0,27$ & 0,55 & 0,30 \\
Satisfação de mãe & 0,13 & 0,63 & 0,39 & 0,39 \\
Aliança confiável com o amigo & 0,19 & 0,67 & 0,45 & 0,44 \\
Companhia do pai & 0,14 & 0,69 & 0,48 & 0,47 \\
Vulnerabilidade & $-0,22$ & 0,71 & 0,51 & 0,50 \\
Conflito com pai & $-0,15$ & 0,72 & 0,52 & 0,52 \\
Desajustamento & 0,15 & 0,73 & 0,54 & 0,54 \\
Cuidado do amigo & 0,13 & 0,75 & 0,55 \\
Fuga e esquiva & $-0,16$ & 0,75 & 0,56 \\
Reavaliação positiva & 0,11 & 0,75 & 0,57 & 0,57 \\
Companhia da mãe & 0,11 & 0,76 & 0,58 & \\
\hline
\end{tabular}

Pode-se observar que todos os fatores reunidos compreendem 57\% da variância da variável dependente. Sozinho, o fator depressão (ou seja, a ausência de características depressivas) é responsável por 30\% da variância do aumento da satisfação de vida total dos jovens participantes da pesquisa.

\section{Discussão}

Os dados revelam que os jovens apresentam um bom nível ou um nível moderadamente alto de satisfação de vida (Nickerson \& Nagle, 2004). Os resultados deste estudo corroboraram tais evidências. Detectou-se que a média de satisfação de vida para os jovens participantes, tanto globalmente quanto em suas diferentes dimensões, apresentou-se entre as classificações 3 e 4 , demonstrando estarem entre relativamente e bastante satisfeitos com diferentes aspectos de suas vidas.

Observaram-se, entretanto, algumas diferenças entre gêneros. Novamente, como já descrito na literatura (Nickerson \& Nagle, 2004), as participantes do sexo feminino apresentaram médias mais altas no fator Escola. Elas mostraram-se bastante satisfeitas com as atividades escolares e com outros aspectos, como relação com professores e colegas. A média de respostas dos participantes do sexo masculino descreveu-os como relativamente satisfeitos com essas questões.

No que se refere às relações estabelecidas pelos jovens, neste estudo optou-se por apresentar apenas os achados relativos às diferenças que foram significativas entre sexos, pois pesquisas prévias demonstram que gênero é uma variável que exerce importante influência no desenvolvimento de vínculos durante essa fase da vida (Cordeiro, 2006; Wong \& Csikszentmihalyi, 1994).

Os resultados demonstraram que as meninas parecem valorizar mais a relação com amigos do que os meninos. Outras pesquisas já haviam trazido resultados semelhantes, indicando que meninas possuem um nível maior de intimidade com seus pares e melhores amigos (Cordeiro, 2006).

Nesta pesquisa, meninos apresentaram médias mais altas para o fator Conflito com o Melhor Amigo. Wong e Csikszentmihaly (1994) trazem indícios de que jovens do sexo masculino relacionam características de agressividade a um maior poder de dominância, enquanto para meninas esse poder estaria relacionado à capacidade de estabelecer vínculos. Esses resultados fazem sentido quando compreendemos que, provavelmente, ao avaliar uma relação como satisfatória (como observado nas respostas dadas pelas jovens em relação a seus melhores amigos), dificilmente essa mesma relação poderia ser caracterizada como conflituosa. Dessa forma, a satisfação na relação com amigos deve ocorrer no sentido inverso dos conflitos com esses mesmos pares.

No que se refere à relação com os pais, pode-se perceber que os participantes do sexo masculino apresentaram médias significativamente mais altas nos fatores auxílio instrumental do pai e satisfação na relação com o pai. Com relação aos dois primeiros, as médias foram mais próximas da classificação 4, o que significa que eles necessitam muitas vezes do auxílio do pai para 
realizar determinadas tarefas e que estão muito satisfeitos com essa relação. Apesar de essas médias terem sido mais altas entre os jovens do sexo masculino, a classificação dada pelas jovens para os mesmos fatores variou entre 3 e 4, o que significaria que elas necessitam seguidamente do auxílio do pai e estão muito satisfeitas na relação com ele.

Do mesmo modo, observou-se que os participantes do sexo masculino apresentaram médias significativamente mais altas para o fator revelação/intimidade com o pai. Aqui também se constata que, apesar da diferença entre médias, tanto para o sexo masculino quanto para o feminino essas médias tendem para a classificação 2, que significa que apenas algumas vezes os jovens sentem-se abertos a uma relação de intimidade com o pai. Entretanto, percebe-se que a mãe, para ambos os sexos, é a figura parental mais escolhida para o estabelecimento de relações de intimidade.

A avaliação das estratégias de coping utilizadas pelos participantes demonstra que, entre os fatores que agrupam tais estratégias, não houve uma variação muito grande das médias. Percebe-se que as médias variaram entre 1,04 (desvio-padrão de 0,61) para a utilização da estratégia de fuga-esquiva e 1,37 (desvio-padrão de 0,69) para a de suporte social. Deve-se levar em conta o fato de o inventário ser respondido por meio de uma escala Likert, com alternativas de classificação de 0 a 3, em que 0 significa que a pessoa não utilizou a estratégia, 1, usou um pouco, 2, usou bastante e 3, usou em grande quantidade. Em função de muitas pessoas que não utilizaram determinada estratégia terem marcado zero, as médias ficaram baixas. Desse modo, entende-se que as médias de todos os fatores mostram-se mais perto de 1, ou seja, em média os participantes de ambos os sexos utilizaram "um pouco" cada um dos grupos de estratégias de coping.

Antes de preencherem o inventário, os participantes deveriam descrever uma situação pela qual passaram e que considerassem estressante. A partir de tal descrição deveriam classificar em qual intensidade utilizaram as estratégias para lidar com o problema. Foi bastante ampla a gama de situações descritas pelos jovens, tendo sido citadas tanto situações mais corriqueiras como problemas relacionados com a escola (como tirar nota baixa, discutir com um colega ou pro22 fessor) ou trabalho e problemas com os pais, até situações mais extremas como uso de drogas, envolvimento com tráfico de drogas ou violência familiar. Observou-se que as situações corriqueiras relacionadas à família e à escola e trabalho foram as mais presentes. Por essa razão, possivelmente, as médias para a utilização de estratégias para lidar com os problemas não tenham sido altas. Além disso, durante as aplicações, pode-se perceber que muitos jovens tiveram dificuldades em se lembrar de uma situação para descrever, o que pode ter gerado um maior distanciamento entre o que foi lembrado e o que realmente foi vivido. O fato de nenhuma estratégia ter se sobressaído em relação às outras também pode significar que esses adolescentes contam com um leque mais variado de estratégias e que eles poderiam lançar mão de qualquer uma delas, de acordo com o tipo de problema enfrentado.

Apesar de não terem sido encontradas diferenças significativas, constatou-se que as jovens apresentaram a média mais alta, dentre todos os fatores do inventário de coping, para o de suporte social, enquanto os jovens apresentaram a média mais alta para o fator aceitação de responsabilidade. A pesquisa de Arteche, Bandeira e Gozalvo (2003) encontrou diferenças significativas entre os sexos para os mesmos fatores no mesmo sentido.

Dentre as características de personalidade acessadas por meio da EFN, não se observou diferença significativa entre os sexos para as médias do fator Vulnerabilidade. Essas médias ficaram dentro de percentis classificados como médio para a amostra normativa do instrumento (Hutz \& Nunes, 2001). Da mesma forma, as médias obtidas para os jovens e as jovens participantes deste estudo no fator Ansiedade enquadraram-se dentro dos percentis médios daquela amostra. Contudo, observou-se uma diferença significativamente mais alta nesse fator para os participantes do sexo feminino. Esse resultado é semelhante ao obtido pela amostra normatizada do EFN, em que as mulheres apresentaram uma média de 75,65 (desvio-padrão de 23,13) e os homens, de 68,93 (desvio-padrão de 22,55).

Nos fatores desajustamento e depressão, por sua vez, a amostra apresentou médias classificadas em um percentil alto, de acordo com o grupo normativo da escala. Em ambos os fatores, os participantes do sexo masculino apresentaram médias mais altas do que as jovens, mesma característica encontrada na população estudada por Hutze Nunes (2001). 
As explicações para as diferenças encontradas entre os jovens participantes desta pesquisa e o grupo normativo podem residir no fato de que a amostra de Hutz e Nunes (2001) conta com universitários cuja maioria se encontra em uma faixa mais ampla de idade: entre 18 e 22 anos. Os participantes deste estudo são estudantes de escolas estaduais com idades entre $14 \mathrm{e}$ 23 anos, com uma média de idade de 16,6 anos (desvio-padrão de 1,4 anos). Sendo assim, percebe-se que enquanto o grupo normativo encontra-se finalizando a adolescência, os participantes desta pesquisa estão no auge dessa fase, período que pode ser entendido como muito mais crítico e conflituoso, em que problemas de desajustamento e sentimentos depressivos são mais comuns (Monteiro \& Lage, 2007).

Na análise de regressão múltipla, a satisfação de vida foi considerada como a variável dependente, e relações com pais e melhor amigo(a), estratégias de coping e traços de personalidade como variáveis independentes. Partiu-se da hipótese de que tais variáveis exerceriam influência sobre a satisfação de vida dos jovens participantes.

Os resultados indicaram que dos 47 fatores referentes a todos os instrumentos considerados como variáveis independentes (incluindo-se aqui também as variáveis sexo e idade), 11 predisseram a satisfação de vida. Os achados desta pesquisa revelaram que o fator depressão do EFN foi a variável que melhor predisse a satisfação de vida neste grupo. A quinta e a sétima variável que melhor explicaram satisfação de vida foram os fatores vulnerabilidade e desajustamento, que também fazem parte da escala de neuroticismo. É importante ressaltar que para os três fatores a correlação estabelecida com a variável satisfação de vida foi negativa, ou seja, a ausência ou diminuição de tais características contribuem para que os jovens sintam-se mais satisfeitos com suas vidas. Esses dados nos remetem a estudos anteriores que consideram traços de personalidade, especialmente a extroversão e o neuroticismo, como fortes preditores da satisfação de vida (Diener \& Lucas, 1999).

O segundo, o quarto, o sexto e o último fator que explicaram o nível de satisfação de vida dos jovens foram os fatores do inventário de rede de relações denominados satisfação na relação com a mãe, conflito com o pai (no caso, ausência de conflito) e companhia da mãe respectivamente. Questões vinculadas à relação estabelecida entre jovens e seus pares também apareceram como importantes na predição da satisfação de vida. Os resultados demonstram que os fatores aliança confiável e relação de cuidado com o melhor amigo estão, respectivamente, em terceiro e oitavo lugar.

Pesquisadores relatam que outro fator que exerce influência sobre o bem-estar de adolescentes é o suporte social de pais e amigos, sendo que a presença de um suporte positivo se correlaciona negativamente com problemas emocionais e de desajustamento (Huebner, 2004). Confiança e comunicação entre os jovens, seus pais e seus pares também se mostram relacionados ao grau de satisfação experienciado (Nickerson \& Nagle, 2004). Na fase da adolescência, há uma mudança da natureza do mundo social das pessoas, aumentando a importância dos pares do mesmo sexo e os laços de amizade. No início dessa fase, os pares passam a ser percebidos como companhias mais importantes do que os pais. Porém, mesmo com a importância dada ao desenvolvimento da intimidade entre os adolescentes e seus amigos, pesquisadores afirmam que os pais continuam sendo importantes, permanecendo como uma fonte de apoio significativa (Buhrmester \& Furman, 1987). Nesta pesquisa, os resultados demonstram o que foi explicitado pelos estudos anteriores. Apesar de a amizade exercer um papel importante nessa fase da vida em termos de satisfação de vida para os jovens, a relação com os pais não perde seu grau de significado.

Por fim, percebe-se que apenas dois dos oito fatores do Inventário de Coping aparecem como variáveis relevantes na predição da satisfação de vida dos participantes. Esses são os fatores de fuga-esquiva (que conta com itens como: "fiz como se nada tivesse acontecido") e a reavaliação positiva (é composto por itens como: "mudei ou cresci como pessoa de uma maneira positiva"). Neste estudo, enquanto o fator de fuga-esquiva estabelece uma correlação negativa com a satisfação de vida, o fator reavaliação positiva constitui uma correlação positiva com a variável dependente.

Nesta pesquisa, optou-se por utilizar como diretriz de avaliação da efetividade das estratégias de coping a proposta de Aldwin e Reverson (1987), que relaciona coping efetivo com aspectos relacionados à saúde física e mental. Desse modo, poderíamos concluir que, para 
os jovens desta pesquisa, o uso de estratégias de reavaliação positiva e o não uso de estratégias de fuga-esquiva estariam relacionados a uma satisfação de vida alta. Esses resultados podem ser comparados aos obtidos por Williams e De Lisi (1999), que relatam que as estratégias de coping tendem a variar com os avanços cognitivos, e que adolescentes mais velhos utilizariam um número mais frequente de estratégias que lidam diretamente com o problema, como a Reavaliação Positiva. Desse modo, parece que as estratégias que lidam mais diretamente com o problema poderiam se classificadas como mais maduras e, neste estudo, como mais eficazes para a obtenção de um maior nível de satisfação de vida. Além disso, estudos anteriores também apontam para o fato de estratégias como as de fuga-esquiva e evitação serem desadaptativas (Schmidt, Dell'Aglio \& Bosa, 2007) e relacionadas a uma diminuição do nível do bem-estar psicológico (Câmara \& Carlotto, 2007).

\section{Considerações Finais}

Por meio do presente estudo foi possível obter um perfil de uma amostra aleatória por conglomerados de jovens estudantes do E.M. de escolas estaduais de Porto Alegre no que se refere à satisfação de vida, relações estabelecidas com pessoas significativas (pais e melhores amigos), estratégias de coping utilizadas para enfrentar certos eventos de vida e características de personalidade.

Os resultados desta pesquisa são importantes por contar com um grande número de participantes e, dessa forma, contribuir para o conhecimento de fatores relacionados à adolescência normal no Brasil. Como limitação do estudo salienta-se o fato de os instrumentos contarem com muitos itens, o que pode ter sido bastante cansativo para os participantes.

Sugere-se, para a continuidade e ampliação da presente pesquisa, a realização de outros estudos que contemplem uma faixa etária mais distribuída de adolescentes e jovens, incluindo-se também estudantes de outros estados e instituições privadas. Estes estudos seriam importantes para um maior conhecimento acerca do desenvolvimento de adolescentes e jovens na

24 realidade brasileira.

\section{Referências}

Associação Brasileira de Empresas de Pesquisa. (2003). Critério de classificação econômica Brasil da Associação Brasileira de Empresas de Pesquisa. Recuperado em março 12, 2006, disponível em http://www.abep.org

Aldwin, C. M., \& Revenson, T. A. (1987). Does coping help? A reexamination of the relation between coping and mental health. Journal of Personality and Social Psychology, 53 (2), 337-348.

Arteche, A. X. (2003). O Impacto do trabalho nas variáveis coping e bem-estar subjetivo em uma amostra de adolescentes. Dissertação de mestrado não-publicada, Universidade Federal do Rio Grande do Sul, Porto Alegre.

Arteche, A., Bandeira, D. R., \& Gozalvo, I. S. (2003). Projetos sociais profissionalizantes: repercussões nas estratégias de coping e no bem-estar subjetivo de participantes adolescentes. Revista de Psicologia da Universidade do Contestado, 1 (1), 5-14.

Bardin, L. (1979). Análise de conteúdo. São Paulo: Edições 70.

Buhrmester, D., \& Furman, W. (1987). The development of companionship and intimacy. Child Development, 58 (4), 1101-1113.

Câmara, S. G., \& Carlotto, M. S. (2007). Coping e gênero em adolescentes. Psicologia em Estudo, 12 (1), 87-93.

Cordeiro, R. A. (2006). Aparência física e amizade íntima na adolescência: estudo num contexto pré-universitário. Análise Psicológica, 24 (4), 509-517.

Diener, E., \& Lucas, R. (1999) Personality and subjective well-being. In D. Kahneman, E. Diener \& N. Schwarz (Eds.), Well-being: the foundations of hedonic psychology (pp.213-229). New York: Russel Sage Fundation.

Feist, G. J., Bodner, T. E., Jacobs, J. F., Miles, M., \& Tan, V. (1995). Integrating top-down and botton-up structural models of subjective well-being: a longitudinal investigation. Journal of Personality and Social Psychology, 68 (1), 138-150.

Folkman, S., \& Lazarus, R. S. (1985). If it changes it must be a process: study of emotion and coping during three stages of a college examination. Journal of Personality and Social Psychology, 48 (1), 150-170.

Furman, W., \& Buhrmester, D. (1992). Age and sex differences in perceptions of networks of personal relationships. Child Development, 63 (1), 103-115.

Giacomoni, C. H., \&Hutz, C. S. (2008). Escala multidimensional de satisfação de vida para crianças: estudos de construção e validação. Estudos de Psicologia (Campinas), 25 (1), 23-35. doi: 10.1590/S0103-166X2008000100003.

Hayes, N., \& Joseph, S. (2003). Big 5 correlates of three measures of subjective well-being. Personality and Individual Differences, 34 (4), 723-727.

Huebner, E. S. (1991). Initial development of the student's life satisfaction scale. School Psychology International, 12 (3), 231-240.

Huebner, E. S. (2004). Research on assessment of life satisfaction of children and adolescents. Social Indicators Research, 66 (1), 3-33. 
Hutz, C. S., \& Nunes, C. H. S. (2001). Escala fatorial de ajustamento emocional/neuroticismo-EFN.São Paulo: Casa do Psicólogo.

Lazarus, R., \& Folkman, S. (1984). Stress, appraisal and coping. New York: Springer Publishing.

Marques, J. C. (1996). Estilos de relações interpessoais na adolescência. Psico, 27 (1), 47-57.

Marques, J. C., \& Horn, K. R. V. (2002). Relações interpessoais em pré-adolescentes, adolescentes e universitários brasileiros: um estudo transcultural. Psico, 33 (2), 245-272.

Monteiro, K. C. C., \& Lage, A. M. V. (2007). A depressão na adolescência. Psicologia em Estudo, 12 (2), 257-265.

Nickerson, A. B., \& Nagle, R. J. (2004). The influence of parent and peer attachments on life satisfaction in middle childhood and early adolescence. Social Indicators Research, 66 (1/2), 35-60.

Pavot, W., Diener, E., Colvin, C. R., \& Sandivik, E. (1991). Futher validation of the satisfaction with life scale: evidence for the cross-method convergence of well-being measures. Journal of Personality Assessment, 57 (1), 149-161.

Savóia, M. G., Santana, P. R., \& Mejias, N. P. (1996). Adaptação do inventário de estratégias de coping de Folkman e Lazarus para o português. Psicologia USP, 7(1/2), 183-201.

Schmidt, C., Dell'Aglio, D. D., \& Bosa, C. A. (2007). Estratégias de coping de mães de portadores de autismo: lidando com dificuldades e com a emoção. Psicologia: Reflexão e Crítica, 21 (1), 124-131.

Williams, K., \& De Lisi, A. M. (1999). Coping strategies in adolescents. Journal of Applied Developmental Psychology, $20(4), 537-549$.

Wong, M. M., \& Csikszentmihalyi, M. (1994). Affiliation motivation and daily experience: Some issues on gender differences. Journal of Personality and Social Psychology, $60(1), 154-164$

Recebido em: 14/11/2008

Versão final reapresentada em: 9/2/2010

Aprovado em: 13/7/2010 